\title{
Self-Efficacy as a Predictor of Patient-Reported Outcomes in Adults with Congenital Heart Disease
}

\author{
Corina Thomet $^{1,2}$, Philip Moons ${ }^{2,3}$, Markus Schwerzmann ${ }^{1}$, Silke \\ Apers $^{2}$, Koen Luyckx ${ }^{4}$, Erwin N. Oechslin ${ }^{5}$, Adrienne H. Kovacs ${ }^{5,6}$
}

${ }^{1}$ Center for Congenital Heart Disease, Department of Cardiology, Inselspital, Bern University Hospital, University of Bern, Bern, Switzerland. E-mail: corina.thomet@insel.ch, markus.schwerzmann@insel.ch

${ }^{2}$ Department of Public Health and Primary Care, KU Leuven - University of Leuven, Leuven, Belgium. E-mail: Philip.moons@kuleuven.be, silke.apers@kuleuven.be

${ }^{3}$ Institute of Health and Care Science, University of Gothenburg, Gothenburg, Sweden

${ }^{4}$ School Psychology and Child and Adolescent Development, KU Leuven - University of Leuven, Leuven, Belgium. E-mail: koen.luyckx@kuleuven.be

${ }^{5}$ Toronto Congenital Cardiac Centre for Adults, Peter Munk Cardiac Centre, University Health Network and University of Toronto, Toronto, Canada. E-mail: erwin.oechslin@uhn.ca

${ }^{6}$ The Knight Cardiovascular Institute, Oregon Health \& Science University, Portland, Oregon, United States. E-mail: kovacs@ ohsu.edu

All authors take responsibility for all aspects of the reliability and freedom from bias of the data presented and the discussed interpretation.

Corresponding author:

Corina Thomet, $\mathrm{MScN}$

Center for Congenital Heart Disease

Inselspital, Bern University Hospital

University of Bern

3010 Bern, Switzerland

Tel. +41316323657

Fax: +41316328050

E-Mail: corina.thomet@insel.ch

Conflicts of interest: None 


\section{Abstract}

Objective: Self-efficacy is a known predictor of patient-reported outcomes (PROs) in individuals with acquired diseases. With an overall objective of better understanding PROs in adults with congenital heart disease (CHD), this study aimed to: (i) assess self-efficacy in adults with CHD, (ii) explore potential demographic and medical correlates of self-efficacy, and (iii) determine whether self-efficacy explains additional variance in PROs above and beyond known predictors.

Methods: As part of a large cross-sectional international multi-site study (APPROACH-IS), we enrolled 454 adults (median age 32 years, range: 18-81) with CHD in two tertiary care centers in Canada and Switzerland. Self-efficacy was measured using the General SelfEfficacy (GSE) scale, which produces a total score ranging from 10 to 40 . Variance in the following PROs was assessed: perceived health status, psychological functioning, health behaviors, and quality of life (QOL). Hierarchical multivariable linear regression analysis was performed.

Results: Patients' mean GSE score was $30.1 \pm 3.3$ (range: 10 - 40). Lower GSE was associated with female sex $(\mathrm{p}=0.025)$, not having a job $(\mathrm{p}=0.001)$, and poorer functional class $(\mathrm{p}=0.048)$. GSE positively predicted health status and QOL, and negatively predicted symptoms of anxiety and depression, with an additional explained variance up to $13.6 \%$. No associations between self-efficacy and health behaviors were found.

Conclusions: GSE adds considerably to our understanding of PROs in adults with CHD. Given that self-efficacy is a modifiable psychosocial factor, it may be an important focus for interventions targeting CHD patients' well-being.

Keywords: Self Efficacy; Patient-Reported Outcomes; Heart Defects, Congenital; Multicenter Study 


\section{Introduction}

In developed countries, adults with congenital heart disease (CHD) now outnumber children with CHD. ${ }^{1}$ Despite significant medical and surgical improvements, CHD remains a life-long medical condition and long-term complications are common. ${ }^{2}$ Living with CHD often entails physical and psychosocial adaptations to the underlying defect and its treatment and also has lifestyle implications with respect to physical activity, employment, and family planning. Thus, optimizing patients' overall well-being and quality of life (QOL) has emerged as an important goal when caring for adults with CHD. ${ }^{3}$ To achieve this aim, patient-reported outcomes (PROs) are increasingly valued in addition to traditional medical parameters. ${ }^{4}$ PROs are directly reported by patients, "without interpretation of the patient's response by a clinician or anyone else". ${ }^{5}$ PROs thus offer unique insight into how patients sense and judge their health and wellbeing.

A large international study investigating PROs in CHD was performed: Assessment of Patterns of Patient-Reported Outcomes in Adults with Congenital Heart disease - International Study (APPROACH-IS). ${ }^{6}$ APPROACH-IS demonstrated that PROs were independently and consistently predicted by functional status, age, and unemployment status. ${ }^{7}$ The impact of selfefficacy as a potential explanatory factor above and beyond other known predictors is unknown. Self-efficacy refers to beliefs about one's ability to perform desired tasks; individuals with higher self-efficacy "approach difficult tasks as challenges to be mastered rather than as threats to be avoided". 8

Self-efficacy has previously been linked with PROs. In patients with non-cardiac conditions (e.g., breast and gastro-intestinal cancer), low self-efficacy has been associated with increased symptom severity, more pain and decreased physical and emotional functioning. ${ }^{9}$ Following myocardial infarction, patients who rated the perceived consequence of the disease higher also reported low general self-efficacy. ${ }^{10}$ Among adolescents and adults with other chronic illnesses, such as diabetes or chronic heart failure, higher self-efficacy has been associated with better 
QOL. ${ }^{11,12}$ A recent published study also found higher transition readiness in adolescent CHD patients with greater self-efficacy. ${ }^{13}$ Self-efficacy has not yet been evaluated in adults with CHD, and it is unclear to what extent self-efficacy predicts PRO above and beyond known demographic and clinical correlates.

Therefore, the aims of the current study were to 1) assess self-efficacy in adults with CHD, 2) explore potential demographic and medical correlates of self-efficacy, and 3) investigate whether self-efficacy explains additional variance in PROs above and beyond known predictors.

\section{Methods}

The present study is a sub-study conducted within APPROACH-IS, which was a cross-sectional multicenter study of PROs in more than 4000 adults with CHD from 24 centers in 15 countries (ClinicalTrials.gov: NCT02150603). ${ }^{6,14}$ For the current sub-study, two participating centers (Toronto, Canada and Bern, Switzerland) included an additional assessment of self-efficacy within their set of questionnaires. Approval for APPROACH-IS, including this sub-study, was obtained by the corresponding local ethic committees, according to the principles outlined in the Declaration of Helsinki.

\section{Study population and procedure}

Inclusion criteria were consistent with the APPROACH-IS protocol: (i) CHD diagnosed before the age of 10 years, (ii) at least 18 years old at the time of study participation, (iii) physical, cognitive and language skills to complete the study questionnaires, and iv) cardiology followup at one of the two participating centers. ${ }^{6}$ Exclusion criteria were: (i) prior heart transplantation and (ii) primary pulmonary hypertension. Data collection took place between January and December 2014. At the Toronto center, patients were recruited during clinic visits; survey completion (in English) occurred in the clinic setting or at home (in which case patients were 
given stamped, pre-addressed envelopes). At the Bern center, patients were mailed a questionnaire package with a consent form (available in either German or French) and a stamped self-addressed envelope prior to their scheduled follow-up appointment. Where no translation was already available, forward-backward procedures based on the MAPI method ${ }^{15}$ to ensure that cross-cultural, conceptual and linguistic equivalence was ensured.

\section{Measures}

\section{Demographic and medical characteristics}

Study participants completed a demographic survey that included age, sex, marital and parenting status, education and employment status, and religiosity. Patients' functional status was assessed with the NYHA functional class I-IV self-report questionnaire, in which class I indicates no limitations and class IV indicates severe limitations with symptoms even while at rest. ${ }^{6}$ Medical records were consulted to document defect complexity (categorized as mild, moderate or great), ${ }^{16}$ history of cardiac surgery, history of congestive heart failure, arrhythmias, implantation of a pacemaker or implantable cardioverter defibrillator, and documented mood or anxiety disorders.

\section{General self-efficacy}

Self-efficacy was measured with the 10-item General Self-Efficacy (GSE) scale. ${ }^{17,}{ }^{18}$ An example of an item is "I am confident that I could deal efficiently with unexpected events." Responses are provided on a four-point scale ranging from 1 ("not at all true") to 4 ("exactly true") and summed to a total score ranging from 10 to 40. A higher total score reflects a higher level of self-efficacy. This scale has been used in various settings with internal consistency coefficients ranging from 0.75 to $0.91 .{ }^{19}$ In the present study, the corresponding internal consistency coefficient was 0.90 , indicating strong reliability. 


\section{Patient Reported Outcomes (PROs)}

Self-report questionnaires were used to assess four PRO domains: perceived health status, health behaviors, psychological functioning and QOL. ${ }^{6}$ Perceived health status was assessed with the EuroQOL-5D Visual Analog Scale ${ }^{20}$ and the 12-item Short Form Health Survey. ${ }^{21}$ Scores on the latter instrument produce a physical component summary (PCS) as well as a mental component summary (MCS). Health enhancing and health compromising behaviors were measured with the Health-Behavior Scale-Congenital Heart Disease. ${ }^{22}$ Psychological functioning was assessed using the Hospital Anxiety and Depression Scale ${ }^{23}$, which produces an anxiety score (HADS-A) and depression score (HADS-D). Quality of life was appraised using the Linear Analog Scale (LAS-QOL) ${ }^{24}$ and the Satisfaction with Life Scale (SWLS). ${ }^{25}$ Online Table 1 provides an expanded definition of the domains as applied in APPROACH-IS as well as the interpretation of scores, validity and reliability of the above mentioned measurements.

\section{Statistical analysis}

For categorical variables, absolute numbers and percentages are presented. Continuous variables are presented as means and standard deviations if normally distributed, or medians with range, if not normally distributed. To investigate whether GSE differed according to demographic and medical characteristics, unpaired t-tests or ANOVA and Mann-Whitney Utest or Kruskal Wallis tests were computed as appropriate. To assess how PROs are affected by patients' self-efficacy, we conducted hierarchical, multivariable linear regression analyses. We ran separate analyses using the following dependent variables: perceived health status (Physical Component Summary (PCS), Mental Component Summary (MCS) and the EuroQol 5 Dimensions-Visual Analog Scale (EQ-VAS)), health behavior (CHD Health-Behavior scale), psychological functioning (Hospital Anxiety and Depression scale (HADS-A, HADS-D)) and QOL (LAS-QOL and SWLS). Using a hierarchical approach, in the first step we included demographic and clinical variables, namely, functional NYHA class, age and employment 
status, because these variables have been identified previously as correlates of PROs within the APPROACH-IS dataset ${ }^{7}$. Further we included sex, marital status, educational level, center (Bern vs Toronto) and disease complexity. In the second step we included general self-efficacy, which allowed us to investigate a change in explained variance. In all analyses, the null hypothesis was rejected for p-values $<0.05$ and two-sided tests were used. Statistical analysis was performed using IBM SPSS Statistics 21 (Armonk, NY: IBM Corp.).

\section{Results}

\section{Patient characteristics}

Of the 809 eligible patients approached by mail or in person, $454(56 \%)$ agreed to participate and completed questionnaires. The majority of participants (80\%) had CHD of moderate or great complexity. Most patients (58\%) reported being in NYHA class I. A total of $365(80 \%)$ patients had undergone cardiac repair in the past, half of them were operated in 2001 or later. Additional demographic and medical characteristics are summarized in table 1.

[Insert Table 1]

\section{General self-efficacy scores and its correlates}

The mean GSE score was $30.1 \pm 3.3$ (range: $10-40$ ). Univariate analysis showed that GSE scores were significantly lower among women $(\mathrm{p}=0.025)$, in patients who were unemployed, job seeking or on disability ( $\mathrm{p}=0.001)$, or in NYHA Class III or IV $(\mathrm{p}=0.048)$ (Table 2). GSE scores did not differ as a function of age, marital status, children, educational background, or religiosity. There was also no association between GSE scores and the following medical characteristics: CHD complexity, history of congestive heart failure, history of arrhythmias, having a cardiac device or documented mood or anxiety disorders. When performing multivariable linear regression analysis, only gender and NYHA Class turned out to be significant correlates of GSE.

[Insert Table 2] 


\section{The relationship between self-efficacy and PRO}

Multivariable linear regression analysis was conducted to assess whether general self-efficacy adds a significant portion of the explained variance of PROs. Results indicated that after adjusting for demographic and clinical factors, higher GSE significantly predicted better health status (PCS, MCS and EQ-VAS), less anxiety and depression (HADS-A and HADS-D), and better QOL (LAS QOL and SWLS). However, self-efficacy was not related to CHD-specific health behaviors. In Table 3, we separately present the $\mathrm{R}^{2}$ variance explained by the overall model (i.e., including variables from both steps) as well as the change in $\mathrm{R}^{2}$ variance after the second step in which GSE was entered. As shown in this table, the additional variance associated with the inclusion of GSE in the model was over $10 \%$ for MCS, HADS-D, and SWLS.

[Insert Table 3]

\section{Discussion}

Although self-efficacy had been associated with QOL and other PROs in patients with other chronic conditions, its relevance for adults with CHD was previously unknown. Therefore, this study was undertaken to understand demographic and medical correlates of self-efficacy and investigate whether self-efficacy explains variance in PROs above and beyond known demographic and clinical predictors.

\section{General self-efficacy in adults with CHD}

The mean GSE score of adults with CHD in this study was 30.1, which is similar to the mean score of 29.5 that has been observed in the international general population. ${ }^{26}$ Schwarzer \& Jerusalem, the authors of the GSE scale, do not recommend categorizing in high and low selfefficacy. This suggests that, as a group, their self-efficacy is comparable to the general population. Perhaps this should not be surprising, given that most have mastered multiple health-related challenges throughout their lives. 
Correlates of self-efficacy observed in the present study were consistent with findings of previous studies in diverse chronic patient populations. ${ }^{27-29}$ In our patient cohort, lower selfefficacy was observed in patients who were female and not employed. We additionally found that lower self-efficacy was observed in patients with poorer functional status, but did not differ as a function of disease complexity. Although causality cannot be determined from a crosssectional study, providers should remain aware that self-efficacy is likely more affected by the symptoms and experiences of patients rather than the original CHD diagnosis.

\section{Self-efficacy as predictor of PROs}

We observed that self-efficacy contributed significantly to the explained variance of PROs beyond that attributable to demographic and clinical factors. Most notably, after including GSE scores, the explained variance increased when predicting depressive symptoms (13.6\%), mental health status (10.7\%), satisfaction with life (10.9\%), QOL (9.2\%), and anxiety symptoms (5.9\%). Thus, self-efficacy is a factor that warrants significant attention as clinicians and researchers strive to better understand and enhance the lives of adults with CHD. It is interesting that self-efficacy added very little $(0.9 \%)$ to the model predicting physical health status. However, this might be due to the fact that the explained variance in the overall model predicting physical health status was the highest of all PROs (almost 55\%) after the first step, before self-efficacy was entered into the model.

Surprisingly, we did not observe a relationship between self-efficacy and health behaviors. This was unexpected because numerous studies previously demonstrated that self-efficacy is associated with better health behaviors such as adhering with dietary or physical activity recommendations. ${ }^{30-32}$ Among children and adolescents with CHD, the belief in one's selfefficacy was shown to correlate with increased physical activity, independent of disease complexity. ${ }^{33,34}$ This discrepancy with our results may be related to the fact that we used a general self-efficacy measure, instead of an exercise-related questionnaire as was administered in pediatric studies. As the GSE questionnaire was developed to measure a confidence in 
managing general adverse situations, it may thus be less applicable to adherence with specific health behaviors.

Our results regarding the links between self-efficacy with mental health status, anxiety and depression are consistent with observations in healthy samples as well as patients with chronic disease. ${ }^{35,36,37}$ A meta-analysis of 181 studies among cancer patients demonstrated large effect sizes for (i) the positive relationship between QOL and self-efficacy for coping with cancer, and (ii) the inverse relationship between distress and self-efficacy for coping with cancer. ${ }^{38}$ It should be acknowledged, however, that the directionality of the relationships between selfefficacy and PROs remains unknown from our study. It is plausible that higher self-efficacy throughout the life of an adult with CHD leads to a sense of general accomplishment and thus overall well-being. ${ }^{8}$ It is equally plausible that better psychological health leads one to be more optimistic that their attempts to master tasks (both related and unrelated to health) will be successful.

In summary, the recognition of the significant relationship between general self-efficacy and PROs holds important implications for interventions targeting mental health of adults with CHD. For example, a multidisciplinary self-management course based on Bandura's selfefficacy concept has shown to be effective in increasing self-efficacy for individuals with chronic conditions, ${ }^{39}$ including arthritis, diabetes and heart diseases. The program included strategies on decision making, problem solving skills and social persuasion through group sessions. Participants not only showed better self-efficacy and health status (pain, depression, fatigue) after one year, but also better communication with health care providers and fewer visits to the emergency department. This example is consistent with other studies on selfmanagement programs based on Bandura's self-efficacy concept. ${ }^{40}$

General clinical recommendations for nurses and other healthcare professionals involved in the care of adults with CHD can therefore be adapted from existing self-management guidelines: establishing a reliable and collaborative relationship, engaging in goal-setting to 
help patients achieve their goals (both related and unrelated to health), and asking patients about their confidence in making changes. ${ }^{41}$ During regular clinical encounters in the inpatient or outpatient setting, nurses can focus on enabling patients by fully engaging them in decisionmaking and highlighting patient achievements. Examples warranting positive verbal reinforcement include attending clinic appointments despite logistical challenges, educating themselves about their diagnoses, and effectively explaining symptoms to providers. Some patients may also benefit from peer support groups. ${ }^{42}$

\section{Methodological considerations}

A strength of this study is that it was undertaken within a large international study with a sound research methodology based on established conceptual foundations. Further, by including patient cohorts from two centers in Canada and Switzerland, we avoided single-center bias and are thus more confident in our ability to explore the relationship between general self-efficacy and PROs. However, there are also methodological limitations to be considered when interpreting the data. The first pertains to the generalizability of study findings. We cannot conclude that our data are applicable to adults with CHD who are not followed in a major center or who are followed at our centers but chose not to take part in our study. The same refers to different ethnic groups, since the majority of our study population was Caucasian/white. Second, the cross-sectional design does not allow us to determine directionality of the relationships between self-efficacy and PROs. To investigate this, longitudinal data or an intervention targeting a change in self-efficacy would be required. Cross-lagged analyses on longitudinal data would allow us to determine if self-efficacy is influencing PROs, or vice versa. Furthermore, future studies on self-efficacy enhancing interventions are important to learn whether increasing self-efficacy yields improvements of PROs. Third, we did not measure disease-specific or behavior-specific self-efficacy, which might have contributed to the absence of a relationship between self-efficacy and health behaviors. Fourth, history of heart-failure and 
arrhythmias as well as documentation of anxiety and depression was abstracted from the medical record and objective verification was not possible.

Finally, although statistically significant relationships between general self-efficacy with sex, employment status, and functional class were observed, the absolute differences were small. It would be important to define the minimal clinically important difference for the GSE scale in order to determine whether these differences are clinically meaningful.

\section{Conclusions}

Higher self-efficacy was associated with more desirable scores in several PRO domains, notably mental health status, symptoms of depression, and QOL. Given that self-efficacy is a modifiable psychosocial variable, this holds important potential as a target for clinical intervention, both in regular clinical encounters as well as within the context of mental health intervention.

\section{Implications for Practice}

- The patients' confidence in their ability to manage life challenges, was associated with several positive outcomes, particularly mental health and QOL.

- Cardiovascular nurses can play an important role in the assessment of general selfefficacy in patients with CHD.

- Promoting self-management strategies may help to enhance patients' general selfefficacy. 


\section{Acknowledgement:}

The Authors declare that there is no conflict of interest. 


\section{References}

1. Gilboa SM, Devine OJ, Kucik JE, et al. Congenital heart defects in the United States: Estimating the magnitude of the affected population in 2010. Circulation. 2016; 134: 101-9.

2. Moons P, Bovijn L, Budts W, Belmans A and Gewillig M. Temporal trends in survival to adulthood among patients born with congenital heart disease from 1970 to 1992 in Belgium. Circulation. 2010; 122: 2264-72.

3. Bratt EL and Moons P. Forty years of quality-of-life research in congenital heart disease: Temporal trends in conceptual and methodological rigor. Int $J$ Cardiol. 2015; 195: 1-6.

4. Rumsfeld JS, Alexander KP, Goff DC, Jr., et al. Cardiovascular health: The importance of measuring patient-reported health status: A scientific statement from the American Heart Association. Circulation. 2013; 127: 2233-49.

5. Food and Drug Administration. Guidance for industry on patient-reported outcome measures: use in medical product development to support labeling claims. Federal Register, 2009, p. 65132-3.

6. Apers S, Kovacs AH, Luyckx K, et al. Assessment of Patterns of PatientReported Outcomes in Adults with Congenital Heart disease - International Study (APPROACH-IS): rationale, design, and methods. Int J Cardiol. 2015; 179: 334-42.

7. Moons $\mathrm{P}, \mathrm{Kovacs} \mathrm{AH}$, Luyckx $\mathrm{K}$, et al. Patient-reported outcomes in adults with congenital heart disease: Inter-country variation, standard of living and healthcare system factors. Int J Cardiol. 2018; 251: 34-41.

8. Bandura A. Self-efficacy. In: Ramachaudran VS, (ed.). Encyclopedia of human behavior. New York: Academic Press, 1994, p. 71-88.

9. Kelleher SA, Somers TJ, Locklear T, Crosswell AD and Abernethy AP. Using patient reported outcomes in oncology clinical practice. Scand J Pain 2016; 13: 6-11.

10. Lau-Walker M. Relationship between illness representation and self-efficacy. $J$ Adv Nurs. 2004; 48: 216-25.

11. Kristofferzon ML, Lindqvist R and Nilsson A. Relationships between coping, coping resources and quality of life in patients with chronic illness: a pilot study. Scand J Caring Sci. 2011; 25: 476-83.

12. Cramm JM, Strating MM, Roebroeck ME and Nieboer AP. The importance of general self-efficacy for the quality of life of adolescents with chronic conditions. Soc Indic Res. 2013; 113: 551-61.

13. Stewart KT, Chahal N, Kovacs AH, et al. Readiness for transition to adult health care for young adolescents with congenital heart disease. Pediatr Cardiol 2017: 1-9.

14. Apers S, Kovacs AH, Luyckx K, et al. Quality of life of adults with congenital heart disease in 15 countries: Evaluating country-specific characteristics. J Am Coll Cardiol. 2016; 67: 2237-45. 
15. Acquadro C, Conway K, Giroudet C and Mear I. Linguistic validation manual for health ourcome assessments. France: Mapi Institute, 2012.

16. Warnes CA, Liberthson R, Danielson GK, et al. Task force 1: The changing profile of congenital heart disease in adult life. J Am Coll Cardiol. 2001; 37: 1170-5.

17. Schwarzer R and Jerusalem M. Generalized Self-Efficacy Scale. In: Weinmann J, Wright S and Johnston M, (eds.). Measures in health psychology: A user's portfolio Causal and control beliefs. Windsor, England: NFER-NELSON, 1995, p. 35-7.

18. Luszczynska A, Scholz U and Schwarzer R. The General Self-efficacy Scale: Multicultural validation studies. J Psychol. 2005; 139: 439-57.

19. Scholz U, Gutiérrez Doña B, Sud S and Schwarzer R. Is general self-efficacy a universal construct? Eur J Psychol Assessment. 2002; 18: 242-51.

20. Group E. EuroQol--a new facility for the measurement of health-related quality of life. Health Policy. 1990; 16: 199-208.

21. Ware JE, Kosinski M, Turner-Bowker DM, Sundaram M, Gandek B and ME. M. User's Manual for the SF-12v2 Health Survey Second Edition. QualityMetric, Incorporated, 2009.

22. Goossens E, Luyckx K, Mommen N, et al. Health risk behaviors in adolescents and emerging adults with congenital heart disease: psychometric properties of the Health Behavior Scale-Congenital Heart Disease. Eur J Cardiovasc Nurs. 2013; 12: 544-57.

23. Zigmond AS and Snaith RP. The hospital anxiety and depression scale. Acta Psychiatr Scand. 1983; 67: 361-70.

24. Moons P, Van Deyk K, De Bleser L, et al. Quality of life and health status in adults with congenital heart disease: a direct comparison with healthy counterparts. Eur J Cardiovasc Prev Rehabil. 2006; 13: 407-13.

25. Diener E, Emmons RA, Larsen RJ and Griffin S. The Satisfaction With Life Scale. J Pers Assess. 1985; 49: 71-5.

26. Hinz A, Schumacher J, Albani C, Schmid G and Brähler E.

Bevölkerungsrepräsentative Normierung der Skala zur Allgemeinen Selbstwirksamkeitserwartung. Diagnostica. 2006; 52: 26-32.

27. Varekamp I, Verbeek JH, de Boer A and van Dijk FJ. Effect of job maintenance training program for employees with chronic disease - a randomized controlled trial on self-efficacy, job satisfaction, and fatigue. Scand J Work Environ Health 2011: 288-97.

28. Gardner JK, McConnell TR, Klinger TA, Herman CP, Hauck CA and Laubach Jr CA. Quality of life and self-efficacy: Gender and diagnoses considerations for management during cardiac rehabilitation. J Cardiopulm Rehabil Prev. 2003; 23: 299-306.

29. Schwarzer R, Bäßler J, Kwiatek P, Schröder K and Zhang JX. The assessment of optimistic self-beliefs: Comparison of the German, Spanish, and Chinese versions of the General Self-efficacy Scale. Applied Psychology. 1997; 46: 6988. 
30. Bandura A. Self-efficacy : the exercise of control. New York: WH Freemann \& Company, 1997.

31. See MT, Chan WC, Huggan PJ, Tay YK and Liaw SY. Effectiveness of a patient education intervention in enhancing the self-efficacy of hospitalized patients to recognize and report acute deteriorating conditions. Patient Educ Couns. 2014; 97: 122-7.

32. Hoffman AJ. Enhancing self-efficacy for optimized patient outcomes through the theory of symptom self-management. Cancer Nurs. 2013; 36: E16-26.

33. Bar-Mor G, Bar-Tal Y, Krulik T and Zeevi B. Self-efficacy and physical activity in adolescents with trivial mild or moderate congenital cardiac malformations. Cardiol Young 2000; 10: 561-6.

34. Ray TD and Henry K. Self-efficacy and physical activity in children with congenital heart disease: Is there a relationship? J Spec Pediatr Nurs. 2011; 16: 105-12.

35. Mystakidou K, Tsilika E, Parpa E, et al. Relationship of general self-efficacy with anxiety, symptom severity and quality of life in cancer patients before and after radiotherapy treatment. Psychooncology. 2013; 22: 1089-95.

36. Tahmassian K and Jalali Moghadam N. Relationship between self-efficacy and symptoms of anxiety, depression, worry and social avoidance in a normal sample of students. Iran J Psychiatry Behav Sci. 2011; 5: 91-8.

37. Martinez-Calderon J, Zamora-Campos C, Navarro-Ledesma S and LuqueSuarez A. The Role of Self-Efficacy on the Prognosis of Chronic Musculoskeletal Pain: A Systematic Review. J Pain. 2018; 19: 10-34.

38. Chirico A, Lucidi F, Merluzzi T, et al. A meta-analytic review of the relationship of cancer coping self-efficacy with distress and quality of life. Oncotarget. 2017; 8: 36800-11.

39. Lorig KR, Sobel DS, Ritter PL, Laurent D and Hobbs M. Effect of a selfmanagement program on patients with chronic disease. Eff Clin Pract. 2001; 4: 256-62.

40. Marks R, Allegrante JP and Lorig K. A review and synthesis of research evidence for self-efficacy-enhancing interventions for reducing chronic disability: implications for health education practice (part I). Health Promot Pract. 2005; 6: 37-43.

41. Lorig KR and Holman H. Self-management education: History, definition, outcomes, and mechanisms. Ann Behav Med. 2003; 26: 1-7.

42. Parent $\mathrm{N}$ and Fortin $\mathrm{F}$. A randomized, controlled trial of vicarious experience through peer support for male first-time cardiac surgery patients: Impact on anxiety, self-efficacy expectation, and self-reported activity. Heart Lung. 2000; 29: 389-400. 


\section{Figures and Tables}

Table 1. Demographic and medical characteristics

Participants $\mathrm{N}=454$

\begin{tabular}{|c|c|}
\hline Median age in years (range) & $32.0(18-81)$ \\
\hline \multicolumn{2}{|l|}{ Age in groups, $\mathrm{n}(\%)$} \\
\hline $18-25$ & $141(31.1)$ \\
\hline $26-30$ & $71(15.6)$ \\
\hline $31-35$ & $62(13.7)$ \\
\hline $36-40$ & $41(7.0)$ \\
\hline $41-45$ & $32(7.0$ \\
\hline$>45$ & $107(23.6)$ \\
\hline \multicolumn{2}{|l|}{ Center, n $(\%)$} \\
\hline Toronto & $176(38.8)$ \\
\hline Bern & $278(61.2)$ \\
\hline Male sex, n (\%), & $248(54.6)$ \\
\hline \multicolumn{2}{|l|}{ Background $(\mathrm{N}=445), \mathrm{n}(\%)$} \\
\hline White/Caucasian & $372(83.6)$ \\
\hline Asian & $32(7.2)$ \\
\hline Hispanic/Latino & $18(4.0)$ \\
\hline Middle-Eastern/Arabic & $15(3.4)$ \\
\hline Black/African-American & $7(1.6)$ \\
\hline Other & $1(0.2)$ \\
\hline \multicolumn{2}{|l|}{ Marital Status $(\mathrm{N}=450), \mathrm{n}(\%)$} \\
\hline Never married/unmarried & $219(48.7)$ \\
\hline Married or living with a partner & $207(46.0)$ \\
\hline Divorced or widowed & $23(5.1)$ \\
\hline Other & $1(0.2)$ \\
\hline Has at least one child $(\mathrm{N}=448), \mathrm{n}(\%)$ & $154(34.4)$ \\
\hline \multicolumn{2}{|l|}{ Employment $(\mathrm{N}=449), \mathrm{n}(\%)$} \\
\hline Full-time/Part-time & $329(73.3)$ \\
\hline Job seeking, unemployed or disability & $64(14.2)$ \\
\hline Homemaker or retired & $28(6.2)$ \\
\hline Other & $28(6.2)$ \\
\hline \multicolumn{2}{|l|}{ Education $(\mathrm{N}=446), \mathrm{n}(\%)$} \\
\hline High school & $206(46.2)$ \\
\hline College degree & $117(26.2)$ \\
\hline University degree & $107(24.0)$ \\
\hline Less than high school & $16(3.6)$ \\
\hline Consider self as religious or spiritual $(\mathrm{N}=444), \mathrm{n}(\%)$ & $152(34.2)$ \\
\hline
\end{tabular}


Complexity of the heart defect, n (\%)

Mild

$91(20.0)$

Moderate

$221(48.7)$

Great

$142(31.3)$

History of cardiac surgery, n (\%)

$365(80.4)$

Congestive heart failure, $\mathrm{n}(\%)$

Never

$422(93.0)$

Past, not current

$19(4.2)$

Current

$13(2.9)$

Patient-reported functional class $(\mathrm{N}=445), \mathrm{n}(\%)$

NYHA I

$264(59.3)$

NYHA II

$137(30.8)$

NYHA III

$37(8.3)$

NYHA IV

7 (1.6)

Any history of arrhythmia, n (\%)

$134(29.5)$

Cardiac device $(\mathrm{N}=453), \mathrm{n}(\%)$

None

$409(90.3)$

Pacemaker

$35(7.7)$

Implantable Cardioverter Defibrillator

$9(2.0)$

Any history of mood disorder $(\mathrm{N}=453), \mathrm{n}(\%)$

$27(6.0)$

Any history of anxiety disorder, n (\%)

$19(4.2)$ 
Table 2. General Self-Efficacy as a function of univariate demographic and medical predictors

\begin{tabular}{|c|c|c|c|}
\hline & $\begin{array}{l}\text { Mean GSE } \\
( \pm \text { Standard Deviation })\end{array}$ & t/F-Value & $\mathrm{p}$ \\
\hline Sex & & 2.250 & 0.025 \\
\hline Male & $30.6( \pm 4.9)$ & & \\
\hline Female & $29.5( \pm 5.4)$ & & \\
\hline Marital Status & & 2.292 & 0.077 \\
\hline Never married & $29.5( \pm 5.2)$ & & \\
\hline Married or living with a partner & $30.7( \pm 5.0)$ & & \\
\hline Divorced or widowed & $31.2( \pm 5.0)$ & & \\
\hline Other & $34.0( \pm 5.2)$ & & \\
\hline Children & $29.9( \pm 5.1)$ & -1.499 & 0.135 \\
\hline Yes & $30.6( \pm 5.2)$ & & \\
\hline No & & & \\
\hline Education & & 0.503 & 0.680 \\
\hline Less than high school & $30.6( \pm 2.7)$ & & \\
\hline High school & $30.1( \pm 5.4)$ & & \\
\hline College degree & $29.6( \pm 5.4)$ & & \\
\hline University degree & $30.4( \pm 4.7)$ & & \\
\hline Work & & 5.249 & 0.001 \\
\hline Full-time/Part-time & $30.1( \pm 5.1)$ & & \\
\hline Homemaker or retired & $31.5( \pm 3.8)$ & & \\
\hline Job seeking, unemployed or disability & $28.5( \pm 5.7)$ & & \\
\hline Other & $32.8( \pm 4.9)$ & & \\
\hline Religiosity & & -1.935 & 0.054 \\
\hline Yes, I consider myself religious or spiritual & $30.8( \pm 4.8)$ & & \\
\hline No & $29.8( \pm 5.3)$ & & \\
\hline Patient-reported functional class & & 2.653 & 0.048 \\
\hline NYHA I & $30.6( \pm 4.8)$ & & \\
\hline NYHA II & $29.6( \pm 5.6)$ & & \\
\hline NYHA III & $28.4( \pm 5.1)$ & & \\
\hline NYHA IV & $29.0( \pm 2.5)$ & & \\
\hline Complexity of the heart defect & & 2.629 & 0.073 \\
\hline Simple & $31.1( \pm 5.1)$ & & \\
\hline Moderate & $30.1( \pm 4.7)$ & & \\
\hline Great & $29.5( \pm 5.7)$ & & \\
\hline
\end{tabular}


Congestive Heart-failure

Never

$30.1( \pm 5.2)$

Past, not current

Current

$30.2( \pm 4.6)$

$29.7( \pm 6.3)$

History of arrhythmia

Yes

No

$29.8( \pm 5.0)$

$30.2( \pm 5.2)$

Cardiac device

None

Implantable Cardioverter Defibrillator Pacemaker

Mood disorder

Yes

No

Anxiety disorder

Yes

No
$30.2( \pm 5.2)$

$32.4( \pm 5.9)$

$28.7( \pm 4.6)$

1.564

$28.6( \pm 6.1)$

$30.2( \pm 5.1)$

1.505

$28.4( \pm 5.5)$

$30.2( \pm 5.1)$ 
Table 3. Multivariable linear regression analysis with GSE as predictor of PROs, adjusted for patient characteristics $(\mathrm{n}=386)$



\begin{tabular}{|c|c|c|c|c|c|c|c|c|}
\hline B (Standard error) & $0.4(0.1-0.6)^{*}$ & $1.2(0.8-1.4)^{* * *}$ & $0.8(0.6-1.0) * * *$ & $-0.2(-0.2--0.1) * * *$ & $-0.2(-0.3--0.2) * * *$ & $-0.03(-0.3-0.3)$ & $1.0(0.8-1.3) * * *$ & $0.5(0.4-0.6) * * *$ \\
\hline $\mathrm{R}^{2}$ overall model & $55.7 \%$ & $36.9 \%$ & $52.0 \%$ & $30.0 \%$ & $44.8 \%$ & $11.4 \%$ & $49.4 \%$ & $46.3 \%$ \\
\hline$R^{2}$ change for general self-efficacy/ & $0.9 \%$ & $10.7 \%$ & $5.9 \%$ & $5.9 \%$ & $13.6 \%$ & $0.0 \%$ & $9.2 \%$ & $10.9 \%$ \\
\hline
\end{tabular}

\# = reference category; Values in table are Estimates (95\% Confidence Intervals); Color coding refers to significance of estimate; PCS=Physical Component Summary; MCS=Mental Component Summary; EQ-VAS=EuroQol 5 Dimensions-Visual Analog Scale; HADS-A=Hospital Anxiety and Depression Scale - Anxiety; HADS-D= Hospital Anxiety and Depression Scale - Depression; LAS QOL= Linear Analog Scale Quality of Life; SWLS=Satisfaction with Life Scale 